\title{
100 Jahre FMH
}

\section{Die Verbindung der Schweizer Ärztinnen und Ärzte FMH im Janusgesicht der Geschichte}

Hans Heinrich Brunner, Präsident FMH

Du gleichst dem Geist, den Du begreifst, nicht mir.

Goethe, Faust I

Vergangenes historisch artikulieren heisst nicht, es erkennen, "wie es eigentlich gewesen ist». Es heisst, sich einer Erinnerung bemächtigen, wie sie im Augenblick der Gefahr aufblitzt.

Walter Benjamin, Geschichtsphilosophische Thesen

\section{Versuch eines geschichtsphilosophischen Präludiums}

Der altrömische Gott Janus und sein Attribut, der Stock, stehen für Übergang aus der Vergangenheit in die Zukunft. So ist sein Name in den ersten Monat des Jahres eingegangen, das aber - sinnigerweise nach römischer Auffassung mit den Iden des März seinen Anfang nahm.

Das Janusgesicht ist sprichwörtlich mit Ambivalenz behaftet, einer Ambivalenz, die vorerst einmal Ausdruck des Ungewissen angesichts einer unwiederbringlichen Vergangenheit und einer ungewissen $\mathrm{Zu}$ kunft ist. Ambivalenz aber auch der Frage gegenüber, wie sich Gegenwart durch Vergangenheit bzw. ihre Protokollierung, die Geschichtsschreibung, definiert.

Beseelt vom Glauben, Vergangenheit könne ein für allemal so dargestellt werden, wie sie eigentlich gewesen sei, sind grosse Teile der Geschichtsschreibung des 19. und 20. Jahrhunderts dem fatalen Glauben zum Opfer gefallen, Gegenwart lasse sich gleichsam in linearer Fortschreibung einer "objektiv» erfassten Vergangenheit definieren. Diesem unkritischen Ansatz, der, weit hinter Kant zurückfallend, die Bedingtheiten des erkennenden Subjekts ebenso systematisch skotomisiert wie das Zwielichtige überkommener Informationen aus der Vergangenheit, sind ideologische Monster entwachsen, die Abermillionen von Menschen den Tod gebracht haben. Vor allem dann, wenn er als Mottenkiste teleologischer Propaganda dienen musste, reichend von nazistischem Rassenwahn über kommunistischen Histomat bis zur Paläontologie eines Teilhard de Chardin. Ein Ende dieses Wahnes ist nicht abzusehen, wie die deliranten "theoretischen" Fundierungsversuche der neoliberalen Markttheorie zeigen, die sich der gleichen Geschichtsklitterungen bedienen.

Geschichte ist nur möglich, wo sie zu ihrem Objekt, ihrem Topos, einen dialektischen Bezug schafft, der in unendlichen rekursiven Ansätzen Subjekt und Objekt in ihren Bedingtheiten und Verklammerungen ausleuchtet und die Brücke zu Sach- und Wahrheits- gehalt von Geschichte leistet. Goethe hat dies geahnt, Walter Benjamin in unerreichbarer Weitsicht und stilistischer Klarheit zu Papier gebracht. Walter Benjamin, der Kommentator des "Flaneurs", des durch Passagen streifenden Flaneurs, der den Bezug zwischen geschichtlichem Ereignis und Gegenwart plötzlich «sieht».

\section{Fortschrittsglaube als Aporie menschlichen Denkens, dem tiefgründige Veränderungen in den Wissenschaften verschlossen bleibt}

Der Übergang vom 19. zum 20. Jahrhundert ist in seinen Tiefen vom Verlust Gottes als sinngebende und zukunftsweisende oberste Instanz gekennzeichnet. Friedrich Nietzsche steht für diesen Umbruch, den er, einem hochsensitiven Seismographen gleich, in Sätzen eingängig wie Hammerschläge aufgefangen hat. Dieser Umbruch vollzieht sich aber wie immer gleichsam in der vollen Breite eines Flusstales, entsprechend vielseitig, vielschichtig, mäandrierend. Dieser Fluss hat gleichsam das Bett vorbereitet für ein immer mächtiger anschwellendes Gewässer namens Fortschrittsglauben, der als geschichtsmächtige Allegorie Wissenschaft und Gesellschaft, insbesondere Politik in einem Ausmasse erfasst hat, das kritisch zu reflektieren vielen noch heute unmöglich ist. Es war vor allem dieser ungebremste, euphorische Fortschrittsglaube, der am 15. Dezember 1901 in Olten zur Gründung einer schweizerischen Ärztekammer geführt hat. Nicht Interessens- oder Einkommenspolitik, nicht KUVG, das damals politisch schon intensiv diskutiert wurde, nicht Schutz vor Interventionen des Staates, der Versicherer oder anderer Gruppen. Den pathetischen Worten des Gründungspräsidenten Dr. Hafter steht nur der unerschütterliche Glaube Pate, eine wissenschaftliche Medizin werde Krankheiten in einem linear aus der Vergangenheit in die Zukunft fortschreitenden Prozess «besiegen».

Da ist nichts zu spüren vom Umbruch, der gerade in diesen Jahren Mathematik, Physik und Philosophie erfasst hatte. Dies mag umso eher zu entschuldigen sein, als die Auswirkungen dieser Umwälzungen auf die Fundamente der Medizin und eine Medizintheorie auch hundert Jahre später nicht in Andeutungen zu spüren sind, wie auch ein nahezu schon läppischer Fortschrittsglaube immer noch ungezählte Hirne von Ärztinnen und Ärzten unausgesetzt vernebelt. So wäre denn ein erster, nicht ermunternder Befund dieser ersten hundert Jahre FMH: Medizin ist ein methodisch-epistemiologisches, periodisch durch steril-realitätsfremde Ethikdiskussionen aufgekräuseltes Brackwasser geblieben.

\section{Geschichtlicher Streifzug durch die Welt an der Schwelle zum 20. Jahrhundert}

Bevor wir näher auf die Frage eintreten, was sich denn in den vergangenen hundert Jahren geändert habe, lohnt sich ein kurzer Streifzug durch die Welt und die Schweiz des Jahres 1901. In oberflächlicher 
Sicht war es für die meisten Menschen der oberen Klassen in Europa und Amerika eine unbeschwerte Phase: Der imperiale Kolonialismus britischer Provenienz war zu historischer Reife gelangt und prägte die Welt; die anderen Grossmächte, oder was sich dafür hielt, versuchten sich auch noch ihr Schnäppchen abzuschneiden, von den Resten Afrikas bis in die pazifischen Eilande. Allgemein war man freihändlerisch und liberal, massvoll wirtschafts- und fortschrittsgläubig. Königin Viktoria und ihre minder glückliche Tochter gleichen Namens, die beide 1901 verstarben, stehen gleichsam als Verkörperungen dieser Ideologien in der historischen Landschaft. Die mit Ausnahme des republikanischen Frankreichs immer noch dominanten Herrscherhäuser, die genetisch, nicht zu ihrem körperlichen Vorteil, mit eben dieser Queen Victoria verbandelt waren, gaben sich adelig und herrschend, waren aber im Grunde viel mehr von der öffentlichen Meinung abhängig, also repräsentativ, als sie selber wahrhaben wollten.

Symbolfigur dieser Zeit war deshalb Wilhelm II, der sich so gerne als den Grossen verewigt gesehen hätte, und dessen unausgeglichenes Temperament sich gelegentlich in geschmacklos-martialischen Ausbrüchen wie der Hunnenrede entlud, der aber den neuen Kunstrichtungen - Matisse stellt 1901 im Salon des Indépendants erstmals seinen neuen dekorativen Malstil aus - und den neuen Wissenschaften gegenüber tolerant war, Bewegungen also, die die geistigen Sprengsätze für die Zerstörung der alten Welt enthielten.

Doch wer wollte sich mit solchen Fisimatenten herumschlagen: Kaiserwetter war's und Europa eine grosse Bühne mit einer nicht enden wollenden Zahl von Manövern, Ausstellungen, Sänger-, Turn- und anderen Festen, und internationale Politik etwas, was scheinbar zum Nachtisch in den Herrscherhäusern ver- und ausgehandelt wurde - was ja auch gut war, solange immer Kaiserwetter herrschte.

Daneben gab es aber ganz andere Welten, die sich häufig nicht einmal bis zur historischen Kenntnisnahme entwickeln konnten: Die ausgebeuteten, darbenden Arbeiterklassen, Millionen von Menschen also, die durch die Industrialisierung jeglichen sozialen Kontext und eigenbestimmte Einkommensstrukturen verloren hatten, was vorsichtig beschönigend als die "soziale Frage» diskutiert wurde, die "armen Heiden in den Tropen", die sich dankbar einem raffinierten Ausbeutungssystem als dem einzigen richtigen Weg zu (einem christlichen) Gott unterwerfen mussten, wobei der gelegentliche Einsatz von Kanonenbooten (der Boxeraufstand in China war ein Jahr zuvor niedergeworfen worden) sich auch spirituell durchaus als hilfreich erwies.

\section{Ärztliche Berufspolitik und geschichtliches Umfeld: uninteressiertes Nebeneinander statt kritischer Reflexion}

Die schriftlichen Dokumente, z.B. das Correspondenzblatt, legen immer wieder Zeugnis von einzelnen Ärzten ab, die sich dieser in den nachfolgenden Jahrzehnten explosiv entladenden Missstände bewusst waren. Für die Gesamtärzteschaft sind diese Ärzte absolut atypisch. Jene hatte sich als Teil der Oberschicht etabliert, was von den Burschenschaften der Universitäten bis in die ärztlichen Gremien lebhaft und ausgedehnt zelebriert wurde. Sowohl für die Gründung vieler kantonaler Gesellschaften wie auch einer schweizerischen Dachorganisation wird der Wunsch nach Pflege der Geselligkeit unter Gleichen, mit deutlicher Spitze gegen andere im Gesundheitswesen Aktive, ausdrücklich und immer wieder als Gründungszweck festgehalten.

Auch die Schweiz durchlebte in dieser Zeit relativ ruhige Jahre: Der Liberalismus war, zunehmend selbst in den Verliererkantonen des Sonderbundskrieges, zum dominierenden Paradigma geworden. Der Interessenausgleich war mit der Umsetzung der Bundesverfassungsrevision von 1874 ein Stück weitergekommen; der die Schweiz heftig erschütternde Kulturkampf war Ende der 80er Jahre abgeebbt, 1891 wurde mit Josef Zemp der erste katholisch-konservative Bundesrat gewählt. Die Wirtschaft war nach den turbulenten Gründerjahren und zum Teil spektakulären Konkursen (z.B. im Bahnensektor) gereift und in ruhigere Fahrwasser gekommen; sie wusste sich im schnell expandierenden Welthandel ihren Platz zu sichern, nicht zuletzt durch eine rasch steigende Zahl von Auslandschweizern, zu denen auch viele Ärzte gehörten, die in allen Teilen der Welt praktizierten und viel zum Renommée der Schweizerischen Medizin im Ausland beigetragen haben. Die deutsche Schweiz war deutschfreundlich, um nicht zu sagen deutschhörig; der schon zitierte Wilhelm II wirkte, propagiert durch eine starke, auch viele Ärzte umfassende deutsche Kolonie, gleichsam als schweizerischer Ersatzmonarch, durchaus nicht zur Freude der Romands, was manche bis in die Gründungsgeschichte der FMH fühlbare Irritationen schuf.

Insgesamt kommen einem diese Jahre wie ein dauerndes Sänger- und Turnerfest entgegen, in denen der neue liberal geprägte Bundesstaat sich zelebrierte und sich eine legitimierende Identität schaffte, die sich keineswegs so nahtlos von den alten Eidgenossen ableitete, wie uns das Pathos dieser Jahre weiszumachen versucht. In diesen Jahren ist eine Grosszahl von historischen Mythen und Mysterien entstanden, die uns die klare Sicht zum Teil bis heute verstellen, getragen von einem Pathos, das bis in die Sitzungen der ärztlichen Vereine zu spüren ist. Die Reden der Präsidenten waren immer erhebend und hochgemut, vor allem aber zukunfts- und fortschrittsgläubig, von den V.H.K. (für Verehrte Herren Kollegen, so einfach war das damals noch) mit Hochrufen "vivat - crescat - floreat" sekundiert.

Sonst finden sich wenig Spuren der aktuellen Politik in der Welt oder der Schweiz in den Dokumenten der Ärztegremien. Ärztliche Tätigkeit bzw. Medizin wurden sehr fokussiert diskutiert; die Gemarkungen der Arbeit am Patienten wurden nur überschritten, wenn es das medizinisch definierte Interesse des Patienten erforderte. Dies zeigt sich exemplarisch, wenn der Centralverein sich zum strafrechtlichen Schutz des Weibes und dessen Ehemündigkeit äussert: Dies hat nach den Worten des Präsidenten Haff- 
ter so zu geschehen, dass «der Boden für Rechtsgrundsätze im Sinne der Physiologie und einer gesunden Ethik vorbereitet wird" (Corr 594). Zivilund Strafrecht werden zum Ausfluss der Physiologie; kritische Reflexionen über die geschichtliche und soziale Bedingtheit von Ethik sind nicht in Ansätzen zu spüren. Das alles hat sich dann auch während 100 Jahren nicht wesentlich geändert.

\section{Gründung der schweizerischen Ärztekammer als Vorläuferin der FMH im Widerstreit zwischen eidgenössischer und kantonaler Politik}

Für das Verständnis des sich fast über ein halbes Jahrhundert hinziehenden Prozesses zur Schaffung einer schweizerischen Ärzteorganisation muss neben dem Umstand, dass der grosse Teil der Ärzteschaft hierfür überhaupt keine Notwendigkeit sah, auf die extrem föderale Struktur der Schweiz, des Gesundheitswesens und auch der ärztlichen Tätigkeit hingewiesen werden. Die einzige überkantonale Phase seiner Biographie durchlebte der Arzt während seines Studiums; auch Ärzte aus Universitätskantonen pflegten damals an ausländischen Universitäten $\mathrm{zu}$ studieren. Mit Praxiseröffnung war auch für Universitätslehrer das Berufsleben kantonal; ausserkantonale Autoritäten wurden nur in der Behandlung wohlhabender Patienten involviert. Gesundheitspolizeiliche Regelungen, Standesordnungen und Honorarordnungen (die Vorläufer der Tarife) waren ausschliesslich kantonal.

Seit Ende des 18. Jahrhunderts hatten sich in den Kantonen Ärztegesellschaften gebildet; in der 2. Hälfte des 19. Jahrhunderts verfügten alle Kantone über kantonale Gesellschaften, die sich in drei überregionalen Ärztegesellschaften zusammengefunden hatten:

- Centralverein, gegründet 1870, umfassend Aargau, beide Appenzell, Baselland, Basel-Stadt, Bern, Glarus, Graubünden, Luzern, Neuenburg, St. Gallen, Schaffhausen, Solothurn, Thurgau, Uri/Schwyz/Unterwalden (urschweizerischer Ärzteverein), Zürich und Zug;

- Société de la Suisse Romande SMSR (gegründet 1867), umfassend Freiburg, Genf, Neuenburg, Waadt und Wallis;

- Società Medica della Svizzera Italiana SMSI mit Tessin.

Interessant an dieser Aufzählung ist, dass die Urkantone gemeinsam auftraten; dies ist nicht als Geringschätzung der eigenen Bedeutung oder Übereinstimmung der Ärzteschaft dieser Kantone in allen Belangen zu interpretieren. Es ist schlichter Ausdruck generellen Desinteresses an schweizerischer Standespolitik und Ausdruck einer Verweigerung gegen alle bundesstaatlichen Interventionen, neben Kostenbewusstsein, das verbietet, Leute für unsinnige Dinge in die Ausserschweiz zu schicken.

Interessant ist auch der Umstand, dass der Kanton Neuenburg Mitglied des Centralvereins sowie der SMSR war und damit eine wichtige Brückenfunktion zwischen Deutsch und Welsch ausübte. Neuenburg war dafür prädestiniert, war es doch erst 1856/57 von den preussischen Souveränitätsansprüchen mit massiver Unterstützung des gesamten Schweizer Volkes freigekommen und mit deutscher und welscher Kultur gleichermassen vertraut.

Damit war die Bühne bereitet für ein Schauspiel in mehreren Akten, das erst 1901 mit der Gründung einer schweizerischen Ärztekammer als Vorläuferin der FMH zum Abschluss kam.

1874 kam es auf Initiative des Centralvereins - die Ärztekommission war ein Gremium dieses Vereins und wurde den Delegierten der anderen Landesteile geöffnet - zur Bildung einer Schweizerischen Ärztekommission aus Ausschussmitgliedern der SMSR, des Centralvereins und, seit 1885, auch der SMSI. Die zugewiesenen Aufgaben gehen aus § 1 der Statuten hervor:

«In Fragen der öffentlichen Gesundheitspflege und des Medizinalwesens die schweizerischen Ärzte vor den Bundesbehörden, wenn nötig auch vor den kantonalen Behörden oder in der Presse vertreten, für Ausführung der von den vereinen erhaltenen Aufträge zu sorgen und die Hülfskasse für Schweizer Ärzte zu verwalten.»

Die Ärztekommission war also eine vorwiegend nach dem Tagsatzungsprinzip auf Mandat der kantonalen und regionalen Ärztegesellschaften funktionierendes Gremium, das hauptsächlich dem Schutz der Ärzteschaft verpflichtet war - eine Funktion, die auch heute noch bei Umfragen unter den Mitgliedern immer noch einsam oben ausschwingt.

Ärztliche Berufspolitik und Bundesstaat:

Ein interessantes Intermezzo

Die Ärztekommission wurde während Jahren von ihrem Präsidenten Sonderegger ausserordentlich stark geprägt, dessen Ceterum censeo war: "Die Medizin muss auch in der Schweiz ein Zweig der öffentlichen Verwaltung werden.» Es ist schwer abzuschätzen, wie weit diese Position von der Basis, insbesondere den Kantonen, überhaupt wahrgenommen oder gar mitvertreten wurde. Wahrscheinlicher ist aufgrund der Akten, dass diese Position überhaupt nicht wahrgenommen bzw. aus kantonaler Sicht schlicht als irrelevant betrachtet wurde.

Der Satz J. L. Sondereggers lässt aber auch erkennen, dass die Organisation des öffentlichen Gesundheitswesens in der Hand der Ärzteschaft lag, die hierfür die Funktion eines Sanitätsreferenten schuf, aus der sich das schweizerische Gesundheitsamt, nachmals Bundesamt für Gesundheitswesen BAG, entwickelte. Dem Erfolg dieser Aktivitäten scheint eine Phase der Inaktivität gefolgt zu sein, während der sich die Ärztekommission schwergewichtig mit der Organisation eines alle drei Jahre stattfindenden Ärztetages beschäftigte. Der Centralverein scheint unter Sonderegger, der Präsident der Ärztekommission und dieses Vereins war, recht dominant gewesen zu sein, nicht immer zur Freude der Ärzte in der lateinischen Schweiz. Der Nachfolger Sondereggers, E. Haffter, war eindeutig auf Ausgleich bedacht und kann als der Architekt der Gründung einer schweizerischen Ärztekammer vor 100 Jahren bezeichnet werden. 
1890-1901: Der lange Gipfelrat zu einem flachen Gipfel namens Ärztekammer

Der Weg war aber garstig und nach etlichen Lawinenniedergängen schwer begehbar gemacht. Der schwerste dieser Niedergänge war die Ablehnung einer Reform der Ärztekommission, deren Zusammensetzung nach einem Modus 1 Delegierter auf 100 Ärzte hätte bestimmt werden sollen. Nach Zustimmung durch die Delegierten wurde die Vorlage durch die SMSR, die schon damals regelmässig in Ouchy tagte, im Herbst 1892 wuchtig verworfen. Die Gründe lassen sich den Dokumenten nicht mit Gewissheit entnehmen; mit einiger Wahrscheinlichkeit war es eine Auflehnung aus dem Bauch gegen die als ungerechtfertigt dominant empfundene Deutschschweiz - auch dies eine nicht ganz unbekannte Konstante schweizerischärztlicher Berufspolitik. Symptomatisch für ein tiefes Misstrauen der Romandie der scheinbar deutschschweizerisch geprägten Ärztekommission gegenüber ist auch der Umstand, dass die welschen Delegierten immer nur für ein Jahr delegiert wurden; offensichtlich war die Angst in der Romandie gross, diese Delegierten könnten sich zu durch alemannischen Ungeist imbibierten Kollaborateuren entwickeln.

Nach dem Wundenlecken kam es erst $1899 \mathrm{zu}$ einem neuen Anlauf, wobei man, aus Erfahrung gewitzigt, auf Änderungen in Funktion und Zusammensetzung der Ärztekommission verzichtete und eine schweizerische Ärztekammer mit proportionaler Delegation aus den Kantonen vorsah. Der Ansatz ist aus politischer wie rechtlicher Sicht bemerkenswert: Hier wurde für eine legislativ wie exekutiv (und selbst richterlich Standesrecht sprechende) Institution ein Parlament - die Ärztekammer - gesucht bzw. geschaffen und diese Ärztekommission, der Vorläufer des Zentralvorstandes, auf eine exekutive Funktion eingeengt. Dies alles nicht aus tiefsinnigen "staatsrechtlichen" Überlegungen, sondern im pragmatischen Bestreben, die Romandie nicht zu provozieren und sie doch einzubinden - eine nicht ganz unbekannte Konstante schweizerisch-ärztlicher Berufspolitik mehr.

\section{Deutschschweizer Dominanz und welsche Verweigerung, eine der vielen Konstanten schweizerischer Ärztepolitik}

Die Initiative lag aber eindeutig beim Centralverein, der auf eine etwas undurchsichtige Weise eine eigene Statutenreform mit einer Initiative zur Gründung einer schweizerischen Ärzteorganisation verband. Er verabschiedete am 28. Oktober 1899 einen Statutenentwurf mit folgenden Schwergewichten:

- Initiativen für Volksgesundheitspflege und Medizinalwesen (die Begriffe werden hier zum ersten Mal getrennt aufgeführt) gegenüber Behörden und Ärztevereinen;

- Vertretung gegenüber Behörden, Presse, wissenschaftlichen Korporationen und Persönlichkeiten des In- und Auslandes (diese hierarchische Demut ist der «Gleichmacherei» des letzten Jahrhunderts definitiv zum Opfer gefallen);

- Ausführung von Aufträgen der kantonalen Ärztegesellschaften;

- Wahrung der Standesinteressen, oberste Instanz bei der Schlichtung von Streitigkeiten unter Ärzten;

- Pflege der Kollegialität - die pointierte Positionierung wie die fast gebetsmühlenartige Wiederholung deuten darauf hin, dass es damit bei uns Ärzten immer etwas gehapert hat - und Organisation der Ärztetage;

- Führung des ärztlichen Hülfsvereins.

Die Zielsetzungen sind mit interessanten Nuancierungen konstant und bleiben dies während der folgenden zwei Jahre. Und noch einmal: Entgegen der später immer wieder vertretenen mystifizierenden Apotheose ex post (Frölich, 1945; Leuch, 1951), mutige Ärzte hätten sich - den Schwurgenossen auf dem Rütli gleich - im Kampf gegen die Lex Forrer bzw. das damals entstehende KUVG zusammengetan, findet sich von dem allem in den Dokumenten nichts. Die organisierte Ärzteschaft scheint die Bedeutung dieser Entwicklungen nicht wahrgenommen $\mathrm{zu}$ haben - eine standespolitische Konstante mehr.

Um so intensiver beschäftigte sie sich - wieder eine Konstante - mit der Frage, ob der Proporz in der geplanten Ärztekammer 1:50 oder 1:100 betragen sollte; man einigte sich auf 1:50, wohl als Entgegenkommen der SMSR gegenüber. Der Versand des entsprechenden Zirkulars an die Kollegen der Romandie verzögerte sich dann ein halbes Jahr, angeblich wegen schwieriger Übersetzungsarbeiten. Als die Post dann endlich in der Romandie angekommen war, löste sie dort - freundlich formuliert und in der Retrospektive verständlicherweise - geringe Begeisterung aus. Sie wurde etwas direkter formuliert als Insult betrachtet und die fehlenden Französischkenntnisse als ebenso lächerliche wie zynische Ausrede. Der Entwurf wurde entsprechend gnadenlos zerzaust; vehement wurde der Vorwurf laut, die Deutschschweiz stelle einmal mehr - die Romandie vor vollendete Tatsachen - wieder eine Konstante. Versuche, einen Ärztetag zur Bereinigung der offenen Fragen einzuberufen, scheiterten ebenso wie die Absicht, in der Sache eine Urabstimmung durchzuführen. Mit dem letzteren Antrag waren ungefähr alle denkbaren kantonalen Sensibilitäten in allen Kantonen wachgerufen.

Am 17. März 1901 wurde dann endlich eine allgemeine Delegiertentagung einberufen, an der sich die Kantone Freiburg, Genf, Wallis und Waadt (nicht aber Neuenburg) einem kantonsweisen Beitritt verweigerten und darauf bestanden, nur als Gruppe beizutreten. Dem Centralverein blieb nichts anderes übrig, als dies zu akzeptieren (1. Juni 1901). Der Weg war damit frei für die Gründung einer Schweizerischen Ärztekammer, gebildet nach einem 1:50-Proporz aus den einzelnen Kantonen der Deutschschweiz und der SMSR als föderativem Verband, dem damals vier welsche Kantone ohne Neuenburg angehörten. 
Die Ärztekommission - bestehend aus neun Mitgliedern, wovon zwei Romands und ein Tessiner - wurde als Exekutive bestellt, die später dann zum Zentralvorstand wurde.

Angesichts der langen und offensichtlich mit vielen Animositäten verbundenen Geburtsgeschichte war die eigentliche, am 15. Dezember 1901 (teilweise, aber weniger verlässlich wird auch der 17 . Dezember 1901 genannt, was aber kaum wahrscheinlich ist, weil sich Ärztinnen und Ärzte noch heute nicht an Dienstagen zu standespolitischem Tun zu versammeln pflegen) im heute nicht mehr existierenden Saal des Restaurants Olten-Hammer unter Leitung des ersten FMH-Präsidenten, E. Haffner, stattfindende Gründungsversammlung eher kühl-geschäftsmässig und entsprechend glanzlos. Dies scheint für die FMH Auflockerungsversuche vereinzelter barock gestimmter Präsidenten ausgenommen - prägend geworden zu sein. Eine 50-Jahr-Feier in grösserem Rahmen wurde vom damaligen Präsidenten Forster als "übertrieben" bezeichnet; die Diskussion drehte sich schliesslich darum, ob man nun der 50 Jahre zu Beginn oder unter Varia einer Ärztekammersitzung gedenken wolle oder gar eine ganztägige Sitzung einberufen müsse, was aber aus finanziellen Gründen als exaltiert rundum abgeschmettert wurde. Den meisten waren "diese Festereien zutiefst zuwider» (von Waldkirch), und so blieb es bei einer halbtägigen Ärztekammer mit einer Vielzahl anderer Geschäfte, einer kurzen Notiz des Präsidenten und einem "offerierten" Mittagessen, dessen Kosten den ZV sehr geschmerzt zu haben scheinen.

\section{Was lernen wir aus dieser Gründungsgeschichte?}

- Geschichte ist auch hier nicht lineare Fortschreibung aus der Vergangenheit in die Zukunft; in dieser Form wird sie nur zu leicht für eigenes Versagen übertünchende Apotheose missbrauchbar, ein Schicksal, wovor sie nur ein kontinuierlicher dialektischer Rückgriff aus möglichst vielen Azimuten bewahrt. Die Ärztekammer wurde nicht gegen die Sozialversicherungsgesetzgebung und für eine freie Arztwahl gegründet; diese Terminologie entstammt späteren Jahrzehnten, ihre Ansätze wurden während langer Zeit schlicht nicht zur Kenntnis genommen bzw. verkannt, ein Versagen, das durch Mystifizierung vergessen gemacht werden sollte.

- Die Geschichte der FMH ist nicht ein von hehren Geistern konsequent verfolgtes hohes Ziel, z.B. der Schaffung ärztlicher Autonomie oder der freien Arztwahl. Ein schweizerisches Ärztegremium wurde während zweier Jahrzehnte als Mandantin des eidgenössischen Bundesstaates gesehen, bevor es als schweizerische Institution zur Interessenvertretung mit minimalen Kompetenzen ausgerüstet gegründet wurde.

- Die Gründungsgeschichte der FMH ist extrem vom Gegensatz von Bundeskompetenzen gegenüber regionalen bzw. föderativen-föderalen Inter- essen geprägt. Der für den neuen Bundesstaat so typische hochgemute Gründungsoptimismus stösst auf statisch-beharrende Kräfte, die zuletzt nur eine minimale Bundeslösung zuliessen.

- Thematiken und Strukturen einer Organisation sind auch in einer Zeit des akzelerierten Wandels bemerkenswert konstant und änderungsresistent: Die föderative Struktur der FMH mit drei regionalen Ärztegesellschaften hat auch die letzte Statutenrevision de facto überlebt, die ZV-Zusammensetzung gehorcht den gleichen Regeln wie vor hundert Jahren und der Röstigraben existiert immer noch, auch wenn er vor 100 Jahren diesen Namen noch nicht trug.

\section{Also nichts Neues unter der Sonne?}

Diese in keiner Weise wertenden Feststellungen sind relevant für die zwei Themen, denen wir uns zum Abschluss zuwenden wollen. In der Tat ist die Frage offen, ob sich denn nicht auch Strukturen, Probleme und Themen geändert hätten. Hier könnte man nun des langen und breiten über Aus-, Weiter- und Fortbildung, über Standesrecht, Ethik, Tarife und anderes mehr sprechen, aber auch Aspekte einer molekularbiologischen Medizin und der Wahrnehmung der mentalen und ökonomischen Grenzen der Medizin.

Die nachfolgenden Zeilen seien aber zwei anderen, keineswegs aber weniger nachhaltigen Paradigmatawechseln gewidmet:

- dem Verlust zünftischer ärztlicher Autonomie und

- der Wandlung der ärztlichen Leistung vom Kunstwerk zum Produkt der modernen Massenfertigung.

\section{Verlust der ärztlichen Autonomie: Vom gelehrten Medicus zum proletarischen Heloten?}

In der Soziologie existiert eine reichhaltige Literatur zu Macht, Wirkung und Zukunft berufsständischer Organisationen bzw. Zünften. Sie ist mit den Namen Max Weber, Parsons, Caplow, Hughes, in neuerer Zeit vor allem aber auch Freidson und Krause assoziiert. Konsens besteht darüber, dass sich diese berufsständischen Organisationen seit dem sogenannten Hohen Mittelalter in Europa entlang den dominierenden Handelsstrassen, vor allem Niederlande/DeutschlandSchweiz-Lombardei, gebildet haben und konstitutiv durch folgende Merkmale gekennzeichnet sind:

- Kontrolle über die vertretenen Berufsleute hinsichtlich ihrer Arbeitsbedingungen und ihrer Marktpositionen inkl. Preisgestaltung durch eine idealerweise autonome Organisation;

- eigener ethischer Kodex ("Standesordnung»), eigene Gerichtsbarkeit;

- Autonomie in der Ausbildung der Mitglieder, für akademische Berufe auch in Form von mit Berufsleuten besetzten Fakultäten;

- Zuständigkeit für ein bestimmtes Gebiet ohne staatliche oder sonstige Intervention. 
Eine kurze Analyse zeigt, dass diese Essentialia einer Standesorganisation in allen Punkten verschwunden oder zumindest massiv bedroht sind:

- Die Kontrolle über die Arbeitsbedingungen sind den Ärzteorganisationen zu einem wesentlichen Teil entglitten, vor allem für Ärzte in Anstellung (Spitäler). Die angestellten Ärzte haben sich wegen meist äusserst divergierender Interessen nicht zu starken Verhandlungspartnern emporschwingen können. Die Motivation hierzu hält sich bei den oberen Kader in Grenzen, weil ein Gärtchen namens Privatpatienten für Trost und Ausgleich sorgte. Die Vergangenheitsform wurde bewusst gewählt, weil dies - zumindest in der konventionellen Form - de facto schon heute von nur mehr marginaler Bedeutung ist.

- Die eigenständige Preisgestaltung gehört seit fast 80 Jahren der Vergangenheit an; der Umbruch wurde durch die Sozialversicherungstarife gemäss KUVG geschaffen und hat nichts mit TARMED zu tun, der nur die letzte Auflage eines solchen Tarifs ist.

- Standesordnungen existieren wohl; ihre Wirkung beschränkt sich aber über weite Strecken auf die Funktion einer edukativen Ermahnung. Eine über Rechtskraft erwachsende regulatorische Bedeutung kommt ihnen (nicht mehr) zu.

- Der letzte Schlag wurde gegen die Autonomie in der Weiterbildung der Ärztinnen und Ärzte geführt. Bei allem Willen, die Ärzteschaft und ihre Standesorganisation vor allem operationell weiter aktiv sein zu lassen, darf dies nicht darüber hinwegtäuschen, dass die strategische Verantwortung in die Hand des Staates übergegangen ist, eines Staates, der taktisch, operationell und auch finanziell unintelligent handelte, würde er das Know-how und den bürokratischen Perfektionismus dieser Standesorganisation sich nicht zumindest vorläufig zunutze machen - und sich damit nicht gleichzeitig ein sanftes Druckmittel schaffen, das auch in anderen Bereichen angewendet werden kann.

- Die unangefochtene alleinige Zuständigkeit für Gesundheitspflege und Medizin, die den Gründern der Ärztekammer noch eine keiner Erwähnung würdige Selbstverständlichkeit war, würde als Forderung in der Öffentlichkeit auf ungläubiges Staunen stossen. Dort sind Ärztinnen und Ärzte ein sogenannter "Partner» unter vielen, reichend vom staatlichen Gesundheitsobservatorium bis zu den Masseuren, Empowermentspezialisten und Wellnessanbetern.

Diese Entwicklungen sind keineswegs für die Schweiz spezifisch; sie ist allen Ärztinnen und Ärzten in den entwickelten Nationen innerhalb der letzten 50 Jahre widerfahren, in den meisten Ländern mit noch viel einschneidenderen Konsequenzen. Die Gründe für diese Entwicklung sind vielfältig:

- Die Autonomie berufsständischer Organisationen stand immer im Gegensatz zum Kapitalismus, der für seine Entfaltung eine unstrukturierte, ohne
Grenzen behinderte, beliebig bewegliche Masse von Konsumenten und Arbeitnehmern braucht, die beliebig über Preise manipuliert werden können. In Ländern mit früher Durchsetzung des Kapitalismus und geringer berufsständischer Struktur Grossbritannien, Frankreich, skandinavische Länder - waren die Ärzteorganisationen, durch den sogenannten Kriegskapitalismus zum Teil massiv befördert, schon Mitte des letzten Jahrhunderts gleichgeschaltete staatliche Agenturen, in den USA wurde die AMA wie eine Fliege an der Wand zerdrückt. Die Schweiz hat sich nun definitiv auch auf diesen Weg begeben, wo sich der Ständerat durch Aufhebung des Kontrahierungszwanges als willfähriger Erfüllungsgehilfe kapitalistischer Interessen profiliert hat. Sehr früh hat die Ärzteschaft aber eine Form des kapitalistischen Brecheisens erfahren: die systematische, gewollte Ausbildung eines Überangebotes von Ärztinnen und Ärzten, die bereits in den 60er Jahren einsetzte.

- Die Bürokratisierung bzw. Verrechtlichung immer grösserer Teile des medizinischen Alltags hat wesentlich zum Autonomiezerfall ärztlicher Standesorganisationen beigetragen. Besonders dramatisch gestaltet sich diese Entwicklung dort, wo kapitalistische Interessendurchsetzung sich auf eine durch Bürgerrechte wenig kontrollierte Bürokratie abstützen kann, wie dies in der EU der Fall ist. Die europäischen Ärzteorganisationen, vor allem wenn von EUs Gnaden, sind zu von der Bürokratie überhaupt nicht mehr beachteten Schwatzbuden verkommen, wo in grenzenloser Selbstbeweihräucherung und intensiver Nabelschau breitspurig Fussnoten diskutiert und beschlossen werden.

- Drehpunkt für alle diese Entwicklungen ist die vollständige Monetarisierung jeglichen menschlichen Tuns, womit die vollständige Beliebigkeit und Austauschbarkeit auch ärztlicher Tätigkeiten auf dem gemeinsamen Nenner Geld möglich geworden ist. Dies leitet über zu einer weiteren Entwicklung, die hier noch anzusprechen ist: Die ärztliche Tätigkeit als Kunstwerk.

\section{Ärztliche Tätigkeit als Kunstwerk?}

Vor hundert Jahren wurde gekonnte und erfolgreiche ärztliche, vor allem operative, Tätigkeit als Kunstwerk verstanden. Die Fallpräsentationen an den kantonalen oder schweizerischen Ärzteversammlungen, die immer den Grossteil der Zeit beanspruchten und die Standespolitik als Marginalie erscheinen lassen, geben in ihrem individuell-fallbezogenen, narrativen Duktus diese auch von der Öffentlichkeit mit fragloser Selbstverständlichkeit mitgetragene Überzeugung wieder. Oder die im Jahre 1901 in drei Sektionen im Correspondenzblatt für Schweizer Ärzte publizierte Serie «Meine chirurgischen Erfahrungen auf dem Gebiet des Magencarcinoms in den Jahren 1896-1900», die jeden "Fall» und seine Behandlung zum unverwechselbaren Einzelfall werden lässt. Oder 
noch heute: der Bergbauer, der stolz seinen Bauch entblösst und die Narben einer Operation zur Schau stellt, mit der ihm Dr. XY geholfen hat. Da ist beim sogenannt "einfachen" Patienten noch eine Wertschätzung der ärztlichen Tätigkeit als handwerkliches Kunstwerk und Unikat zu verspüren, die bei Medienschaffenden, Politikern, Versicherern verlorengegangen ist - Wiedererweckungserlebnisse bei eigener Krankheit vorbehalten.

In den dreissiger Jahren des vergangenen Jahrhunderts hat Walter Benjamin in seinem Essay «Das Kunstwerk im Zeitalter seiner technischen Reproduzierbarkeit» mit hoher Sensitivität und untrüglichem Scharfblick die Verwandlung des Kunstwerks in ein Produkt der Massenkultur perzipiert; seine Ausführungen sind mutatis mutandis auch auf die ärztliche Tätigkeit anwendbar: Ein Kunstwerk ist durch sein einmaliges Dasein an dem Orte, an dem es sich befindet, definiert; dadurch ist auch seine Echtheit definiert. Es ist zum Beispiel das einen Patienten grundsätzlich prägende ärztliche Gespräch, dem solche singuläre Bedeutung zukommt. Das Kunstwerk ist damit nach Benjamin über seine stofflichen Gegebenheiten hinaus durch eine Aura gekennzeichnet, die nur am Ort, am Kunstwerk selbst in einmaliger Weise zum Ausdruck kommt. Die Wahrnehmung eines Kunstwerks ist geschichtlichen Traditionen wie Wandlungen unterworfen, andererseits aber auch an einen Kult, ein Ritual gebunden, das ebenso unverwechselbar-einmalig ist. Medizin hat sich auch als Ritual im Sinne von Benjamin zu vollziehen und kann, weil einmalig, im Grunde nicht tarifarisch abgegolten werden. Auch hier wäre wieder vom selben Bergbauern zu lernen, der noch Fleisch und Butter mitbringt, weil "das Nötli für die Krankenkasse» nicht die wahren Verdienste des Arztes abgelten könne.

\section{Die Beliebigkeit des Leistungserbringers und seiner Tätigkeit}

Das vergangene Jahrhundert hat die Instrumente einer zeitverzugslosen Informationsvermittlung geschaffen und den Ort der "Leistungserbringung" beliebig werden lassen. Damit ist auch der Arzt selbst beliebig und kontingent, seine Arbeit und Leistung standardisierbar, scheinbar messbar, Guidelines unterwerfbar und neudeutsch «benchmarkfähig» geworden. TARMED, medizinische Informatik und speziell Telemedizin, Outcome-orientierte Qualitätssicherung sind aktueller und eklatanter Ausdruck dieses Paradigmenwechsels, der von Medizinalbürokraten aller Schattierungen und Provenienz inkl. managementorientierten Ärztinnen und Ärzten kritiklos zum Götzendienst erhoben wurden. Eine scheinbar konservativ-rückwärtsgewandte Resistenz findet sich bei einzelnen Fachdisziplinen - Psychiaterinnen/Psychiatern und operativ Tätigen allzumal - und bei vielen Patientinnen und Patienten. Eine beharrliche und nachhaltige Resistenz, die zu kritischer Reflexion und Neuorientierung nicht nur einlädt, sondern zwingt.
Diese Jubiläumsreflexionen sind zweifelsohne Essig in den von allen vollmundig gewünschten Festtagswein. Sie können für sich immerhin in Anspruch nehmen, in einer Tradition nüchterner, schnörkelloser Berufspolitik zu stehen, die die meisten meiner Vorgänger ebenfalls zu ihrer Maxime gemacht haben - und damit zum Abschluss auch die Möglichkeit der Frage nach dem "Was tun" gestatten, weil sie auf die Aporie einer Apotheose verzichtet haben.

Ärztliche Standespolitik wird sich in den nächsten Jahren auf das Wiedergewinnen von Autonomie konzentrieren müssen. Dies bedeutet konkret:

- Besetzen von Feldern, die zukünftig von Bedeutung sein werden, wie ärztliche Qualitätssicherungspolitik, Schaffen von relevanten Datenstrukturen in den Händen der Ärztinnen und Ärzte, Gestalten des ärztlichen Berufsfeldes, reichend von praktischen Hilfen und Fortbildung à la carte im ärztlichen Alltag bis zu Massnahmen des Arbeitskampfes, Einbezug von hochqualifizierten, klinisch tätigen Ärztinnen und Ärzten in die Standespolitik, Aufbau von ärztlichen Entscheidungsmechanismen für den Einsatz immer knapper werdender Ressourcen.

- Verabschiedung aus sogenannten «Partnerschaften", die schon lange keine mehr sind, z.B. mit Versicherern, gewissen staatlichen Organisationen oder der immer virtueller wirkenden Front sogenannt liberaler und blauer Berufe. Dies soll nicht inhaltlich wie zeitlich klar begrenzte Zweckallianzen verhindern.

- Verzicht auf nicht zu gewinnende Nachhutgefechte, z.B. den Kampf um hundertprozentig gerechte Arzttarife. Ganz abgesehen davon, dass dieser Krieg schon vor vielen Jahrzehnten mit der Einbindung der Ärzte in die Sozialversicherung verloren wurde, werden Arzttarife klassischen $\mathrm{Zu}$ schnitts mit Wegfall des Kontrahierungszwanges zu historischer Makulatur werden.

- Dafür Konzentration auf Verwirklichung neuer Ideen in der Versorgung unserer Patienten, für die es in Managed-Care-Organisationen, in Hausärzte- oder sonstigen Ärztesystemen ausserordentlich interessante Ansätze gibt. Dies, verbunden mit ärztlicher Kompetenz, erhält die immer wieder geäusserte positive Einschätzung der Ärztinnen und Ärzte durch die Bevölkerung, die unsere sichersten Verbündeten im Kampf um die Sonderstellung ärztlichen Tuns und damit ärztlicher Autonomie sind.

Mein Vorgänger Dr. Hafter hat am 15. Dezember 1901 der Ärztekammer ein kräftiges "Vivat, crescat, floreat» im Dienste von Wissenschaft und Vaterland zugerufen. Wir sind da inhaltlich und formal etwas zurückhaltender geworden: Möge es uns gelingen, der nächsten Generation von Ärztinnen und Ärzten ein sie befriedigendes Berufsfeld zu erhalten, das ihnen gestattet, in voller beruflicher Autonomie nur der Patientin und dem Patienten verpflichtet zu arbeiten und sich als würdige Ärztinnen und Ärzte zu verwirklichen. 